\title{
Effects of shadowing on prose comprehension and problem solving
}

\author{
RICHARD E. MAYER and LINDA K. COOK \\ University of California, Santa Barbara, California 93106
}

\begin{abstract}
In two experiments, subjects listened to a text about radar while either shadowing or not shadowing the passage. Results of Experiment 1 produced a pattern in which both groups performed at similar levels on retention of single facts, the nonshadowers excelled on transfer of the presented information to novel problem solving, and shadowers excelled on verbatim recognition of words. In Experiment 2, there was a pattern in which both groups performed at similar levels for recall of specific information, the nonshadowers excelled on recall of conceptual principles, and shadowers excelled on recall of modifying details. Implications for transfer-appropriate processing and the cognitive capacity hypothesis were examined.
\end{abstract}

When a person is presented new prose information, how does the person's orienting strategy influence what is learned? Recent research using word lists as stimuli has produced two theories: The "levels-of-processing" hypothesis (Craik \& Lockhart, 1972) states that deeper levels of processing (e.g., semantic orienting tasks) during encoding will lead to better performance on memory tests; the "transfer-appropriate processing" hypothesis (Bransford, 1979; Morris, Bransford, \& Franks, 1977; Stein, 1978) states that the type of orienting strategy may influence the type of encoding rather than simply having an overall effect on the strength of memory traces.

This paper investigates the role of the learner's orienting strategy when the to-be-learned materials consist of a technical prose passage rather than a word list. In particular, the text is presented auditorily in chunks, and subjects are asked to engage in one of two orienting strategies: normal listening (nonshadowing group), in which the learner is free to use available cognitive capacity for all comprehension processes, and delayed shadowing (shadowing group), in which the learner repeats each phrase after it is presented. There is some evidence that shadowing reduces the amount of available cognitive capacity (Kerr, 1973). Thus, the goal of this study is to determine the effects of shadowing on the process of comprehension and the outcome of learning.

This paper encourages the development of a theory of prose processing that specifies the role of alternative processing strategies. In particular, the to-be-developed theory must deal with two issues, which can be called

This research was supported by Grant NIE-G-78-0162 from the National Institute of Education and Grant SED77-19875 from the National Science Foundation. Requests for reprints should be sent to either author at the following address: Department of Psychology, University of California, Santa Barbara, California 93106. We gratefully acknowledge the advice and assistance of Jim Voss, Sherman Tyler, and Greg Vesonder in developing a macrostructure and in use of analysis programs. the "cognitive capacity" hypothesis: (1) The cognitive capacity of the comprehender is limited, and (2) there are many information processing activities required in order to comprehend a passage.

Case (1978) notes that the first point, that working memory is limited, was shown by Jacobs (1887) early in the history of experimental psychology. More recently, Kahneman (1973) has shown how "cognitive capacity" can be measured and how it affects comprehension.

Britton and his colleagues (Britton, Holdredge, Curry, \& Westbrook, 1979; Britton, Piha, Davis, \& Wehausen, 1978) have also investigated the role of cognitive capacity in prose processing. For example, Britton et al. (1978) found that when text passages contained test-relevant adjunct questions, the usage of cognitive capacity increased. This increase was measured by recording the response latency to clicks presented during reading, with longer latencies suggesting a higher degree of usage of cognitive capacity. Apparently, adjunct questions encouraged readers to identify and manipulate key concepts in certain portions of the text, and this required additional capacity.

The second part of the cognitive capacity hypothesis, that verbal comprehension can be analyzed as several information processing activities, has been presented by Hunt, Lunneborg, and Lewis (1975, p. 192). These authors suggest that the main information processing activities involved in comprehending a passage are: (1) decoding-the identification of overlearned verbal units such as required in a Posner letter-matching task (Posner, Boies, Eichelman, \& Taylor, 1969), (2) maintenance-the processing and storage of a sequence of verbal units in short-term memory (STM) such as required in a Peterson and Peterson (1959) task, and (3) integration-manipulating and reorganizing information so that it fits with existing knowledge from long-term memory (LTM).

The remainder of this section presents three possible theories of how a reduction in cognitive capacity might 
influence the comprehension process and the outcome of learning in this prose processing task. If shadowing reduces the amount of cognitive capacity (Kerr, 1973), then one or more of the comprehension processesdecoding, maintenance, or integration-may be affected when there is an overload. Since decoding and maintenance are necessary events for all subsequent processes, since all precede integration, and since these are likely to be more automatic than integration, it is plausible that integration would be the first process to be affected if cognitive capacity were overloaded. Therefore, each of the theories described below is based on the idea that shadowing will mainly affect the integration process, that is, the storage of information into LTM. Simon (1976) has suggested that the time to store information in LTM depends on two subprocesses: the time to add the information to memory and the time to modify and elaborate the newly acquired information.

\section{Theory 1: Amount Added to LTM}

The most straightforward idea is that shadowing affects how much information can be transformed (or added) to LTM. This theory predicts that shadowing will mainly hinder the process of adding new information to LTM, and thus, the shadowing group should perform poorer overall than the nonshadowing group on all relevant tests such as recognition, application, recall of details, and recall of underlying principles. This pattern would be reflected in an overall main effect for treatment (shadowing vs. nonshadowing) on tests of remembering the material.

\section{Theory 2: Amount of Elaboration Processing}

A second theory is that shadowing affects how much effort may be given to modifying and elaborating information that is added to LTM. This theory predicts that shadowing and nonshadowing groups will be able to add the same amount of information to LTM, but the shadowing task reduces the amount of elaborative processing on newly added information; thus, performance will be poorer for shadowers than for nonshadowers on tests that depend on a highly elaborated cognitive structure, such as application problems on recall of principles, but there should be no difference between the groups on tasks that do not require highly elaborated structures, such as verbatim recognition or recall of details. This pattern would be reflected in an interaction between treatment (shadowing and nonshadowing) and type of test, in which the interaction is not disordinal (i.e., the lines do not cross).

\section{Theory 3: Type of Elaboration Process}

The third idea is that shadowing affects the type of elaborative processing that is performed on information added to LTM. For example, the shadowers may use a strategy that focuses on retention of information within each presented chunk, whereas nonshadowers may focus on integrating information between chunks. This theory predicts that shadowers will perform worse than nonshadowers on tests that require putting chunks of information together, such as application problems or abstracting out the conceptual theme, but nonshadowers will perform better on tests that require retention within chunks, such as verbatim recognition or recall of marginal details. This pattern would be reflected in an interaction between treatment (shadowing vs. nonshadowing) and type of test, in which the interaction is disordinal (i.e., the lines cross).

\section{EXPERIMENT 1}

In Experiment 1, subjects either silently listened to or shadowed a passage on radar. Learning outcomes were measured by four posttests which varied from verbatim recognition of presented sentences to application of several text principles to solve novel problems.

\section{Method}

Subjects and Design. The subjects were 34 undergraduates from the University of California who participated in order to fulfill a course requirement. None of the subjects reported having previous experience with or knowledge about radar. Seventeen subjects served in the shadowing group, and 17 served in the nonshadowing group. There were four within-subjects measures corresponding to scores on four types of question sets.

Materials and Apparatus. A 640-word tape-recorded passage describing the basic principles of radar was developed from a lengthier treatment by Clarke (1977). The passage avoided unnecessary physics principles and used examples drawn from ordinary experience to explain the phenomena. Table 1 presents a portion of the text. In recording the passage, short pauses were placed between sequences of words corresponding to the natural phrase boundaries within each sentence. This was to ensure that subjects would have both time and an indication of when to repeat what they had just heard. All sequences of words contained no fewer than four and no more than seven words each. The total recorded passage was read at a rate of 70 words/minute in a female voice.

A three-page test booklet was constructed, consisting of four tests. The first sheet contained the syntactic recognition test and lexical recognition test. The syntactic recognition test consisted of five pairs of sentences; one member of each pair was a verbatim sentence from the passage, and the other member of the pair was a syntactically changed version of the same sentence. Syntactically changed statements were formed by taking a sentence from the passage and rearranging sentence parts to form a new sentence that conveyed the same meaning as the original. As an example, the following is a sentence from the passage: "When you think about the returning pulses or echoes you should remember any object in the path of the transmitted beam reflects some of the energy back to the radio receiver." A syntactically changed version of this sentence is as follows: "You should remember, when you think about the reception of the returning pulse or echoes, any object in the path of the transmitted beam reflects some of the energy back to the radio receiver."

The lexical recognition test consisted of five pairs of sentences; one member of each pair was a verbatim sentence from the passage, and the other member of the pair was a lexically changed version of the same sentence. Lexical changes involved 
Table 1

Portion of Radar Text

( 1) Radar means the detection and location of remote objects

( 2) by the reflection of radio waves.

(3) The phenomenon of acoustic echoes is farniliar.

( 4) Sound waves reflected from a building or cliff

(5) are received back at the observer

(6) after a lapse of a short interval.

(7) The effect is similar to you shouting in a canyon

( 8) and seconds later

(9) hearing a nearly exact replication of your voice.

(10) Radar uses exactly the same principle

(11) except that the waves involved are radio waves, not sound waves.

(12) These travel very much faster than sound waves

(13) 186,000 miles per second

(14) and can cover much longer distances.

(15) Thus radar involves simply measuring the time

(16) between transmission of the waves

(17) and their subsequent return or echo

(18) and then converting that to a distance measure.

the substitution of similar words for key words in the sentence For example, the original sentence "When an object is present it leaves a bright spot on the face of the screen" became "When an object is present, it leaves a lightened area on the face of the screen." The five syntactic recognition and five lexical recognition questions were typed onto the same single $8.5 \times 11$ in. sheet of paper, with type of item (syntactic versus lexical) and position of correct answer (top or bottom) randomly ordered. Directions indicated that the subjects were to choose the one of the two paired statements that had come verbatim from the original passage.

The second sheet presented the retention test. This test consisted of 16 questions that assessed the learners' memory of the basic facts presented in the passage. All questions in this set required true-false responses. As an example of these, the text explained that the first models of radar could tell only the speed of an object and not its absolute location and that modern systems now use a map-like screen to find both speed and location. The question assessing knowledge of this information was "Modern types of display systems are able to tell both location and speed of an object while earlier models were only able to tell location," to which the appropriate response was false.

A last sheet contained five application questions. These required conceptual understanding of several text principles that would be applied to novel problem situations not addressed in the text itself, for example: "If an object remained at a constant distance with each measurement, what form would the object's movement take?" or "If you were only able to send out and receive one transmission, what is the most important information you could learn about the object?"

Apparatus included a Panasonic cassette tape recorder and a stereophonic headset. A practice tape was also constructed.

Procedure. Each subject was randomly assigned to either a shadowing or a nonshadowing condition and was tested individually. For the shadowing condition, subjects were told they would be hearing, through stereophonic headphones, a short description of the basic fundamentals of radar. While listening, they were to repeat passage sequences at the pauses provided in the tape. To facilitate familiarity with the type of shadowing required, they practiced on a sequence of six sentences taken from a short story. They were always informed that they would not be responsible for the contents of the practice tape. In the nonshadowing condition, subjects were given the same description of the passage but were told to simply listen carefully through the headphone set. They, however, did not hear the practice tape. Prior to tape presentation, both groups of subjects were told they would be given a test on the passage.

The testing phase occurred immediately after tape presentation. The three test sheets were administered in a counterbalanced order across subjects. Directions to the subject on the top of the syntactic/lexical recognition sheet asked them to pick the one from the pair they thought had come directly from the text. They were encouraged to guess if they were not sure. The directions also explained the two types of possible changes in the sentences. For the retention questions, subjects were directed to think carefully before indicating whether or not the statement was true or false. In the application question set, they were asked to answer each question as thoroughly as possible.

\section{Results and Discussion}

Each answer was scored correct or incorrect, and the percentage correct for each type of test was computed for each subject. A two-way analysis of variance with repeated measures was performed on the data. From the perspective of Theory 1 , it was expected the act of shadowing would reduce the amount of information encoded in LTM. Thus, the shadowing subjects' performance across question sets, regardless of the type of information assessed in each, should have been reduced relative to the nonshadowing condition. The significant main effect for shadowing vs. nonshadowing $[F(1,32)=14.02, p<.001]$ supported this contention. Shadowing subjects correctly answered $68 \%$ of all the question sets, compared with $75 \%$ for the nonshadowing subjects.

There are, however, several pieces of information that cast serious doubt on the adequacy of Theory 1 to account for the obtained data. Table 2 shows the performance of both groups by type of test. First, if shadowing reduced the amount of information encoded, the overall effect noted above should be present for each of the four tests. Yet, a significant interaction between treatment group and type of question $[\mathrm{F}(3,96)=10.21$, $\mathrm{p}<.001$ ] clearly contradicted this prediction. Second, it should be noted that the retention test used in this 
Table 2

Proportion Correct Response by Type of Test for Two Groups (Experiment 1)

\begin{tabular}{lcccc}
$\begin{array}{c}\text { Treatment } \\
\text { Group }\end{array}$ & \multicolumn{2}{c}{ Recognition } & & Reten- \\
Lexical & Syntactic & $\begin{array}{c}\text { Appli- } \\
\text { tion }\end{array}$ & cation \\
\hline Nonshadow & .64 & .70 & .82 & .81 \\
Shadow & .77 & .66 & .79 & .49 \\
\hline
\end{tabular}

Note-Main effect of treatment: $p<.001$; interaction between treatment and type of test: $p<.001$.

study is the test that is most commonly used in prose memory studies. As can be seen, however, there is no evidence to support the prediction that shadowing would reduce the amount of information encoded; the scores on the retention test were not significantly different for the two groups, as measured by a $t$ test $[\mathrm{t}(32)=.25, \mathrm{p}>.20]$. In summary, Theory 1 predicts an overall main effect but no interaction; however, an interaction was obtained. Theory 1 predicts that the overall main effect should be present for the retention test; however, this is not the case. Thus, Theory 1 may be rejected as an adequate explanation of the data. Theories 2 and 3 both are consistent with both of the above facts and thus will now be explored in more detail.

Theory 2 states that shadowing reduces the amount of time available for elaborating on the information, and thus it predicts a pattern of performance in which the shadowers perform worse than the nonshadowers on tests that require an integrated knowledge base (the best example of such tests is the application test), but there should be no differences on tests that do not require a highly elaborated cognitive structure (such as the recognition tests and the retention test). Theory 3 predicts that since the two groups use qualitatively different encoding strategies, the shadowers should perform better on tests that require verbatim memory within a chunk (the best candidate is the lexical recognition test, which requires verbatim memory of one word within one chunk), whereas nonshadowers should perform better on tests requiring integration across chunks (the application test is the best example of integrating across chunks). The general pattern of Treatment by Type of Test interaction cited above and shown in Table 2 is consistent with both predictions. However, in order to more clearly discriminate the two theories, $t$ tests were performed on the differences between the groups for each test. As predicted by both theories, the shadowers performed significantly more poorly than the nonshadowers on the application test $[t(32)=2.49, p<.05]$. Performance for the two groups was not significantly different on the retention test $[\mathrm{t}(32)=.25, \mathrm{p}>.20]$ or on the syntactic recognition test $[\mathrm{t}(32)=.17, \mathrm{p}>.20]$. However, there was only marginal support for Theory 3 , since the shadowers performed marginally better than the nonshadowers on the lexical recognition test $[\mathrm{t}(32)=1.79, \mathrm{p}<.10$ (twotailed test)].
The effects of shadowing can be summarized by noting that (1) shadowing did not seem to reduce the overall amount of information added to memory, since performance on the retention test was not affected, (2) shadowing did seem to reduce the amount of elaborative processing for understanding and integrating chunks together, since performance on the application test was adversely affected, and (3) shadowing may have actually enhanced elaborative processing for verbatim recognition within chunks, since shadowing did positively (albeit only marginally) affect performance on the lexical recognition test. These points are explored further in Experiment 2.

\section{EXPERIMENT 2}

Experiment 2 repeated the shadowing and nonshadowing treatment conditions of Experiment 1 but employed a recall test rather than the four performance tests used in Experiment 1. The results of Experiment 1 indicated that both groups of subjects were equally proficient with regard to learning of specific information, as was assessed by the retention questions. The differential performance on the application questions in Experiment 1 raised the possibility that shadowing precluded the opportunity for structuring an integrated relationship between these individual chunks. Within the passage itself, some of the units of information are more crucial in promoting integration than others. If results parallel those of Experiment 1 (and support Theory 3 ), the following is expected: The nonshadowing group will excel in recall of this type of information, which we call "conceptual idea units," but not in recall of other specific information, which we call "nonconceptual idea units." The differential performance between the groups on lexical recognition questions in Experiment 1 raised the possibility that shadowing enhanced verbatim processing of words within presented chunks. Within the passage itself, each idea unit could be broken down into a central point (such as an "action") and "modifiers" that presented a detail that was not central to the main point of the idea unit. If results parallel those of Experiment 1 (and support Theory 3 ), the shadowing group is expected to excel in recall of these modifying details, but not on recall of the central information.

\section{Method}

Subjects and Design. The subjects were 28 undergraduates from the same population used in Experiment 1 (14 subjects in the shadowing group and 14 subjects in the nonshadowing group).

Materials and Apparatus. The same taped passage, tape recorder, and stereophonic headphones used in Experiment 1 were employed. A response booklet was constructed, which included several sheets of $8.5 \times 11$ in. paper. Directions at the top of the first page asked subjects to write down all they could remember from the tape recording and to organize their recall in a manner that a reader unfamiliar with the phenomena would understand. 
Procedure. Subjects were randomly assigned to either a shadowing or a nonshadowing condition and given the same instructions as in Experiment 1. At the end of the tape presentation, they were given the response booklet and asked to read the printed directions. After the experimenter answered any questions and reemphasized the directions, subjects began their recall.

\section{Results and Discussion}

Protocol scoring. For purposes of protocol scoring, the original passage was broken down into 69 idea units. Each idea unit consisted of a sentence or clause that conveyed one main idea. ${ }^{1}$ A typical passage statement, separated into idea units by slash marks, is the following: "When no object impedes the traveling radar pulse/ it continues its travel/until lost from the screen on the right." Additional examples are given in Table 1. In addition to the 69 idea units, there were eight connectives or introductory comments that were not counted in the analysis.

Each subject's recall protocol was parsed into basic idea units. Then, for each idea unit in the protocol, an attempt was made to match it with one of the 69 idea units in the original text. A match was made if the key words in the protocol idea unit matched the key words in the text idea unit. If a protocol idea unit did not match any of the 69 idea units, it was assigned to one of two alternative categories, inference or summarization. An inference was a new idea unit that was not in the text but was logically related to the text, for example, "The dot's size can be used to determine the size of the object." A summarization was a vague or brief statement about information in the text, such as, "A transmitter sweeps across a certain area." In all cases, the rater was blind to the experimental condition of the subject.

Category analysis. A major question addressed in this study is whether the recall protocols of subjects in the shadowing group differed from those of the nonshadowing group. For example, the results of Experiment 1 suggest that the groups may be equivalent in recall of individual facts (as indicated in the retention tests in Experiment 1) but may differ in recall of conceptual principles needed for transfer (as indicated in the application test in Experiment 1). In order to investigate this idea, the idea units were categorized into two separate sets: conceptual idea units and nonconceptual idea units. Conceptual idea units stated one of the five basic ideas: transmission-a pulse is sent out; reflection-it strikes a remote object; reception-a pulse returns to the source; measurement-the time and direction are recorded; conversion-time may be converted to a measure of distance. The following are examples of conceptual idea units: "Radar means the detection and location of remote objects/by reflection of radio waves. Thus radar involves simply measuring the time/between transmission of the waves/and their subsequent return or echo/ and then converting that to a measure of distance." In addition, other conceptual units described how a transmitter sends radar pulses, how an antenna receives them, and how early models derived distance. The nonconceptual category contained all other idea units, including statements of fact, such as "Radar travels at $186,000 \mathrm{mps}$," descriptions of the parts of radar equipment, and analogies such as tossing a pebble into a pond. There were 20 conceptual idea units and 49 nonconceptual idea units.

Table 3 summarizes the proportion recalled for the groups by category of idea unit. Since protocols contained less than $4 \%$ inferences or summarizations, and since these were evenly distributed between groups, these were not included in any of the analyses reported in this paper. First, a t test was conducted to determine whether the groups differed in overall number of idea units recalled. Although there was a trend for the nonshadowers to perform better than the shadowers, that difference did not reach statistical significance $[t(26)=$ $1.63, \mathrm{p}>.10]$. Thus, if we look only at overall amount recalled in this study, or at overall content retained (retention questions) in Experiment 1, we would conclude that there is no difference in what the two groups learned.

However, since Experiment 1 suggests a pattern of interaction, a two-way analysis of variance was conducted on these data with treatment as a between-subjects factor and type of category as a within-subjects factor. This analysis revealed an interesting interaction between treatment and type of category $[F(1,26)=4.87, p<.05]$, in which the two treatment groups performed at about the same level on nonconceptual idea units but the nonshadowers recalled more than twice as many conceptual idea units. Subsequent $t$ tests indicated that the shadow and nonshadow groups were not significantly different in recall of nonconceptual idea units $[t(26)=.99$, $\mathrm{p}<.20$ ] but that the nonshadowers performed significantly better than the shadowers in recall of conceptual idea units $[t(26)=3.13, p<.01]$.

These results nicely parallel the results of Experiment 1 , in which nonshadowers and shadowers performed at similar levels on retention questions (requiring retention of specific segments of the text) but the nonshadowers performed better on application questions (requiring creative use of the learned materials). These results suggest, but do not directly test, the idea that creative use of information in problem solving depends partly on recall of key conceptual ideas in the passage.

Propositional analysis. The foregoing analysis was

Table 3

Proportion Recalled by Type of Idea Unit for Two Groups (Experiment 2)

\begin{tabular}{ccc}
\hline & & \multicolumn{2}{c}{ Idea Units } \\
\cline { 3 - 3 } $\begin{array}{c}\text { Treatment } \\
\text { Group }\end{array}$ & Conceptual & Nonconceptual \\
\hline Nonshadow & .26 & .29 \\
Shadow & .11 & .24 \\
\hline
\end{tabular}

Note-Interaction between treatment and type of idea unit. $p<.05$. 
based on idea units as the unit of analysis. In the present section, a more "micro" level of analysis is reported, based on Kintsch's "propositional analysis" (Kintsch, 1974; Turner \& Greene, Note 1). Each of the first 32 idea units was further broken down into propositions, with approximately three to six propositions found in each idea unit. For example, the proposition "when no object impedes the traveling radar pulse" would be broken down into the following propositions: (1) IMPEDES (O: pulse; S: no object), (2) QUALITY (when, 1), (3) MODIFY (pulse, radar), (4) MODIFY (pulse, traveling). The recall of each proposition by each of the 28 subjects was determined by rescoring the original protocols.

Each proposition was classified as being a defining part of the idea unit in which it was embedded (these "major" propositions consisted of events such as IMPEDES and of some locations) or as being nonessential to the idea unit (these "minor" idea units consisted of modifiers, qualifiers, conjunctions, and the like). If the nonshadowers attempt to listen for major meaning, they might be less likely to retain minor propositions; if the shadowers have no overall strategy for bringing meaning to the passage, they would be likely to retain equal amounts of major and minor propositions.

Table 4 shows the proportion recalled by type of proposition for two groups. An analysis of variance was conducted with treatment as a between-subjects factor and type of proposition as a within-subjects factor. As can be seen in Table 4, there is an interesting pattern of interaction in which the nonshadowers performed better on major propositions and shadowers performed better on recall of minor propositions $[F(1,26)=13.86, \mathrm{p}<.001]$.

The $t$ tests revealed that the nonshadowers did not perform significantly better than shadowers on recall of major propositions $[\mathrm{t}(26)=1.23, \mathrm{p}>.10]$, but the shadowers did recall significantly more minor details than the nonshadowers did $[\mathrm{t}(26)=2.06, \mathrm{p}<.05]$. This pattern of results is consistent with the pattern in Experiment 1, in which shadowers outperformed nonshadowers on lexical recognition but not on retention. Apparently, the shadowers are more likely to retain surface modifiers and details. For example, shadowers overwhelmingly recall that a pebble is tossed into a "calm" pond, whereas nonshadowers unanimously ignore the adjective. A stepwise discriminant analysis was performed, using score on minor and score on major

Table 4

Mean Number of Propositions Recalled by Type for Two Groups (Experiment 2)

\begin{tabular}{|c|c|c|}
\hline $\begin{array}{c}\text { Treatment } \\
\text { Group }\end{array}$ & $\begin{array}{c}\text { Major } \\
\text {. Propositions }\end{array}$ & $\begin{array}{c}\text { Minor } \\
\text { Propositions }\end{array}$ \\
\hline $\begin{array}{l}\text { Nonshadow } \\
\text { Shadow }\end{array}$ & $\begin{array}{l}8.3 \\
6.1\end{array}$ & $\begin{array}{l}3.4 \\
6.9\end{array}$ \\
\hline
\end{tabular}

Note-Main effect of treatment, n.s.; interaction between treatment and type, $p<.001$. propositions as independent variables. The resulting function relied on only one factor: Minor propositions loaded on the shadowing centroid with a weight of .44 . The function was able to correctly classify 23 of the 28 subjects. Thus, relatively high performance on recall of minor propositions seemed to distinguish most of the shadowers from the nonshadowers.

The foregoing two analyses provided replicatory evidence for the pattern of performance noted in Experiment 1: namely, that shadowers and nonshadowers performed at equivalent levels on tests of memory for basic information, such as retention or recall of nonconceptual idea units, nonshadowers excelled on tests requiring integration of information, such as application problems or recall of the conceptual theme, and shadowers outperformed nonshadowers on tests requiring verbatim memory of details within each chunk of information, such as lexical recognition or recall of minor propositions. These findings are most consistent with Theory 3, which is based on the idea that shadowers and nonshadowers employ qualitatively different elaboration processes and thus acquire qualitatively different learning outcomes. ${ }^{2}$

\section{GENERAL DISCUSSION}

Do subjects who are required to shadow a passage acquire a learning outcome different from that of subjects who just listen to the text? If we had used the traditional measure of overall retention, such as that measured by the retention test of Experiment 1, we would have concluded that there were no major differences between the groups. If we had used the traditional measure of overall amount recalled, such as that measured by total number of propositions recalled in Experiment 2, again we would have found no treatment effect. However, when we look deeper into the performance of our subjects, important differences emerge.

Since the groups manifested different learning outcomes, it is reasonable to suggest that the learning processes were different for the two treatment groups. In this paper, we have outlined three distinct theories of the acquisition process and how it might be affected when cognitive capacity is reduced.

Theory 1 has some similarities to the levels-ofprocessing hypothesis (Craik \& Lockhart, 1972). In particular, Theory 1 states that shadowers have less cognitive capacity available for adding new information to memory; thus, decreasing the capacity available for comprehension processes results in less information being acquired. The levels-of-processing idea is that deeper levels of processing will lead to better performance on memory tests. This proposal is based on research in which recall for a list of words was better if subjects had engaged in semantic orienting tasks during encoding rather than orthographic orienting tasks (Craik \& Tulving, 1975; Hyde \& Jenkins, 1969; Jenkins, 1974). In the present experiments, using a scientific prose passage, there was little evidence for the idea, stated in 
Theory 1 , that shadowing would result in overall decrements in memory test performance: In Experiment 1, there was no significant difference between the groups in retention of basic facts; in Experiment 2, there was no significant difference between the groups in recall of propositions; in both Experiments 1 and 2 there were significant Treatment by Type of Test interactions in which shadowers performed better on some tasks but nonshadowers performed better on others. While these results do not contradict all interpretations on the levels-of-processing idea for prose material, they do clearly allow one to reject a version of levels of processing, as stated in Theory 1.

If the results are highly inconsistent with the predictions of Theory 1 , they are only partially consistent with Theory 2 . Theory 2 suggests that shadowing reduces the cognitive capacity available to perform integrative elaboration among the chunks of acquired information. As predicted, shadowers perform more poorly than nonshadowers on tasks that require integration of information (application problems in Experiment 1 and recall of conceptual idea units in Experiment 2), but not on remembering the basic information (retention problems in Experiment 1 or recall of propositions in Experiment 2). However, the main contradiction to Theory 2 involves cases in which the shadowers actually outperform nonshadowers on tasks requiring a verbatim memory of the details. In Experiment 1, there is only a marginally reliable superiority of shadowers over the nonshadowers on lexical recognition (although the .05 level of significance would be achieved if a one-tailed test were employed); in Experiment 2, the significant superiority of the shadowers over the nonshadowers on recall of minor propositions gives the strongest evidence against Theory 2 . While more research is required, a tentative conclusion is that there is some reason to suspect that Theory 2 may not be able to account for all of the data.

The results of the present study tend to favor Theory 3 , the idea that shadowers and nonshadowers used qualitatively different processes for elaborating on the acquired information. This data is similar in many respects to the concept of transfer-appropriate processing (Bransford, 1979; Morris et al., 1977). For example, Morris et al. (1977) auditorialy presented words and asked some subjects to answer semantic questions for each target word (e.g., does "dog" fit the description “__ has ears?") and other subjects to answer rhyming qucstions for each target word (e.g., does "dog" fit the description "rhymes with log"?). As predicted by the levels-of-processing hypothesis, the semantic group performed better on a test of recognizing that words had been presented in the original list; however, a new finding was that the rhyming group outperformed the semantic group on a test of recognizing which words rhymed with words from the original list. In another study, Stein (1978) visually presented word lists containing words such as "raDio." One group of subjects was asked semantic questions (such as "Does this word use electricity?") and the other group was asked orthographic questions (such as "Does this word have a capital D?"). As in the previous research, the semantic group performed better on recognizing which words had been presented; however, the orthographic group outperformed the semantic group on recognizing which letter in a given word had been capitalized in the original list.

Morris et al. (1977) offer the transfer-appropriate processing hypothesis to account for these results: the idea that orienting strategy may influence the type or quality of encoding rather than its simply having overall effects on the strength of memory traces. In a summary of this line of research, Bransford (1979, p.67) pointed out that "it is no longer profitable to assume that certain items' memory traces are less durable or adequate than others' simply because these items were processed at a shallow level ... and shallower levels of processing can be superior to deeper levels if one designs test situations that assess the kinds of information that actually results from shallower levels of analysis."

Much of the previous work on prose comprehension has involved simple narrative texts or short factual passages. One goal of such research has been to predict probability of recall of text propositions. For example, Kintsch (1974) and Meyer (1975) have developed a case grammar procedure for producing a hierarchical ordering of propositions and have shown that probability of recall of a proposition is related to its level in the hierarchy. Similarly, Rumelhart (1975) and Thorndyke (1977) have developed story grammars for describing the hierarchical relations among events and states in a simple narrative. More recently, Kintsch and van Dijk (1978) have been able to specify a model for learning from text that incorporates assumptions about both case grammar and macrostructure.

The present study extends this work on narratives in two ways. First, we used a scientific text that can be used to generate problem solving. Like Voss and his colleagues (Chiesi, Spilich, \& Voss, 1979; Spilich, Vesonder, Chiesi, \& Voss, 1979), we found that many modifications have to be made to existing techniques when new materials are used, including much larger propositional units and an entirely different macrostructure system.

Second, we used an experimental manipulation to determine whether interfering with cognitive capacity of the subject would influence ability to make use of the macrostructure. Britton's work (Britton et al., 1978; Britton et al., 1979) has demonstrated that manipulations in the prose processing task have measurable effects on cognitive capacity. For example, presence of adjunct questions increases demands on cognitive capacity. Similarly, in the present studies, the nonshadowers were allowed more cognitive capacity to identify and integrate the key conceptual information, whereas the shadowers 
were more likely to attend to and store the surface information as presented.

In the present studies, we manipulated the learner's behavior during prose presentation. However, another way to manipulate cognitive capacity is to identify individual differences. For example, Hunt et al. (1975) found that good readers (i.e., subjects scoring high on tests of verbal ability) differ from poor readers on simple information processing tasks that require holding and manipulating information in memory.

Voss and his colleagues (Chiesi et al., 1979; Spilich et al., 1979) have also investigated individual differences in how subjects listen to an inning of a baseball game. For example, subjects with high knowledge of baseball are more likely than low-knowledge subjects to relate the actions of the game to the goal structure and to maintain important information in working memory. In the present study, our nonshadowers were better able than the shadowers to relate information to five main macrostructure events (transmission, reflection, reception, measurement, conversion).

In an earlier set of experiments, Mayer and Greeno (1972) distinguished between two variables in learning outcomes: Internal connections referred to how well the presented elements were connected with each other in memory, and external connectedness referred to how well the presented elements were connected with an organized body of existing knowledge. In the present examples, we have provided a more specific and detailed examination of the structural differences in what is learned. There is certainly room for more detail and precision, but the present example indicates one way to describe differences in learning from technical prose. One general implication of this work is if the teacher says, "now repeat after me," he or she is insuring that the students will not understand (Glaser, Note 2).

In summary, this work suggests that a better understanding of the limits of cognitive capacity is an important prerequisite to the development of theory of prose comprehension. This work provides some new information that indicates that draining some cognitive capacity (e.g., through shadowing) affects the structure of what is learned and the capacity for creative use of presented information.

\section{REFERENCE NOTES}

1. Turner, A., \& Greene, E. The construction and use of $a$ propositional text base (Tech. Rep. No. 63). Boulder, Colo: Institute for the Study of Intellectual Behavior, 1977.

2. Glaser, R. Personal communication, September 1979.

3. Mayer, R. E., \& Cook, L. Effects of shadowing on prose comprehension and problem solving (Tech. Rep. 80-3). Santa Barbara, Calif: Department of Psychology Technical Report Series in Learning and Cognition, 1980.

\section{REFERENCES}

Bransford, .J. D. Human cognition: Learning, understanding and remembering. Belmont, Calif: Wadsworth, 1979.

Britton, B. K., Holdredge, T. S., Curry, C., \& Westbrook,
R. D. Use of cognitive capacity in reading identical texts with different amounts of discourse level meaning. Journal of Experimental Psychology: Human Learning and Memory, 1979, 5, 262-270.

Britton, B. K., Piha, A., Davis, J., \& Wehausen, E. Reading and cognitive capacity usage: Adjunct question effects. Memory \& Cognition, 1978, 6, 266-273.

CASE, R. Implications of developmental psychology for the design of effective instruction. In A. M. Lesgold, J. W. Pellegrino, S. D. Fokkema, \& R. Glaser (Eds.), Cognitive psychology and instruction. New York: Plenum, 1978.

Chiesi, H. L., Spilich, G. J., \& Voss, J. F. Acquisition of domain-related information in relation to high and low domain knowledge. Journal of Verbal Learning and Verbal Behavior, 1979, 18, 257-273.

CLARKE, D. The encyclopedia of how it works: From abacus to zoom lens. New York: A \& W, 1977.

Craik, F. I. M., \& LockharT, R. S. Levels of processing: A framework for memory research. Journal of Verbal Learning and Verbal Behavior, 1972, 11, 671-684.

Craik, F. I. M., \& Tulving, E. Depth of processing and the retention of words in episodic memory. Journal of Experimental Psychology: General, 1975, 104, 268-294.

Hunt, E., Lunne borg, C., \& Lewis, J. What does it mean to be high verbal? Cognitive Psychology, 1975, 7, 194-227.

Hyde, T. S., \& Jenkins, J. J. Differential effects of incidental tasks on the organization of recall of a list of highly associated words. Journal of Experimental Psychology, 1969, 82, 472-481. $\mathrm{J}_{\text {AcoBs, }} \mathrm{J}$. Experiments on prehension. Mind, 1887, 12, 75-79. JENKINS, J. J. Can we have a theory of meaningful memory? In R. L. Solso (Ed.), Theories in cognitive psychology: The Loyola symposium. Hillsdale, N.J: Erlbaum, 1974.

Kahneman, D. Attention and effort. Englewood Cliffs, N.J: Prentice-Hall, 1973.

Kerr, B. Processing demands during mental operations. Memory \& Cognition, 1973, 1, 401-412.

KINTSCH, W. The representation of meaning in memory. Hillsdale, N.J: Erlbaum, 1974.

Kintsch, W., \& van DiJk, T. A. Toward a model of text comprehension and production. Psychological Review, 1978, 85, 363-394.

Mayer, R. E., \& Greeno, J. G. Structural differences between learning outcomes produced by different instructional methods. Journal of Educational Psychology, 1972, 63, 165-173.

MEYER, B. J. The organization of prose and its effects on memory. Amsterdam: North-Holland, 1975.

Morris, C. D., Bransford, J. D., \& Franks, J. J. Levels of processing versus transfer appropriate processing. Journal of Verbal Learning and Verbal Behavior, 1977, 16, 519-533.

Peterson, L. R., \& Peterson, M. J. Short-term retention of individual verbal items. Journal of Experimental Psychology, 1959, 58, 193-198.

Posner, M. I., Boies, S. J., Eichelman, W. H., \& Taylor, R. L. Retention of visual and name codes of single letters. Journal of Experimental Psychology, 1969, 79, 1-16. (Monograph)

Rumelhart, D. E. Notes on a schema for stories. In D. G. Bobrow \& A. M. Collins (Eds.), Representation and understanding: Studies in cognitive science. New York: Academic Press, 1975.

Simon, H. A. The information storage system called "human memory." In M. R. Rosenweig \& E. L. Bennett (Eds.), Neural mechanisms of learning and memory. Cambridge, Mass: M.I.T. Press, 1976.

Spilich, G. J., Vesonder, G. T., Chiesi, H. L., \& Voss, J. F. Text processing of domain-related information for individuals with high and low domain knowledge. Journal of Verbal Learning and Verbal Behavior, 1979, 18, 275-290.

STEIN, B. S. Depth of processing re-examined: The effects of precision encoding and test appropriateness. Journal of Verbal Learning and Verbal Behavior, 1978, 17, 165-174. 
Thorndyke, P. W. Cognitive structures in comprehension and memory of narrative discourse. Cognitive Psychology, 1977, 9, $77 \cdot 110$.

\section{NOTES}

1. We are using units that are much larger than those in Kintsch's propositions (Kintsch, 1974; Turner \& Greene, Note 1). In this respect, we are consistent with Voss's (Chiesi et al., 1979; Spilich et al., 1979) technique for analyzing real-world prose.
2. Supplemental analyses were carried out in the data from Experiment 2. These include a detailed analysis of the structure of the text and the development of macrostructure representations of the text. A summary of these analyses is available from the authors as part of a technical report (Mayer \& Cook, Note 3 ). These analyses generally provide replicatory support for the findings and conclusions stated above.

(Received for publication March 11, 1980; revision accepted April 15, 1980.) 